ARTICLE

DOI: $10.1038 / s 41467-017-01065-7$

OPEN

\title{
Anomalous quantization trajectory and parity anomaly in Co cluster decorated $\mathrm{BiSbTeSe}_{2}$ nanodevices
}

Shuai Zhang ${ }^{1}$, $\mathrm{Li} \mathrm{Pi}^{2,3}$, Rui Wang ${ }^{1}$, Geliang $\mathrm{Yu}^{1}$, Xing-Chen Pan ${ }^{1}$, Zhongxia Wei ${ }^{1}$, Jinglei Zhang ${ }^{3}$, Chuanying $\mathrm{Xi}^{3}$, Zhanbin Bai ${ }^{1}$, Fucong Fei ${ }^{1}$, Mingyu Wang ${ }^{1}$, Jian Liao ${ }^{4}$, Yongqing $\mathrm{Li}^{4}$, Xuefeng Wang ${ }^{5}$,

Fengqi Song ${ }^{1}$, Yuheng Zhang ${ }^{3}$, Baigeng Wang ${ }^{1}$, Dingyu Xing ${ }^{1} \&$ Guanghou Wang ${ }^{1}$

Dirac Fermions with different helicities exist on the top and bottom surfaces of topological insulators, offering a rare opportunity to break the degeneracy protected by the no-go theorem. Through the application of Co clusters, quantum Hall plateaus were modulated for the topological insulator $\mathrm{BiSbTeSe}_{2}$, allowing an optimized surface transport. Here, using renormalization group flow diagrams, we show the extraction of two sets of converging points in the conductivity tensor space, revealing that the top surface exhibits an anomalous quantization trajectory, while the bottom surface retains the 1/2 quantization. Co clusters are believed to induce a sizeable Zeeman gap ( $>4.8 \mathrm{meV}$ ) through antiferromagnetic exchange coupling, which delays the Landau level hybridization on the top surface for a moderate magnetic field. A quasi-half-integer plateau also appears at -7.2 Tesla. This allows us to study the interesting physics of parity anomaly, and paves the way for further studies simulating exotic particles in condensed matter physics.

\footnotetext{
${ }^{1}$ National Laboratory of Solid State Microstructures, College of Physics and Collaborative Innovation Center of Advanced Microstructures, Nanjing University, Nanjing 210093, China. ${ }^{2}$ Hefei National Laboratory for Physical Sciences at Microscale, University of Science and Technology of China, Hefei 230026, China. ${ }^{3}$ High Magnetic Field Laboratory, Chinese Academy of Sciences and Collaborative Innovation Center of Advanced Microstructures, Hefei, Anhui 230031, China. ${ }^{4}$ Institute of Physics, Chinese Academy of Sciences, Beijing 100190, China. ${ }^{5}$ National Laboratory of Solid State Microstructures, Collaborative Innovation Center of Advanced Microstructures, and School of Electronic Science and Engineering, Nanjing University, Nanjing 210093, China. Shuai Zhang, Li Pi and Rui Wang contributed equally to this work. Correspondence and requests for materials should be addressed to F.S. (email: songfengqi@nju.edu.cn) or to Y.Z. (email: zhangyh@ustc.edu.cn) or to B.W. (email: bgwang@nju.edu.cn)
} 
$\mathrm{n}$ three-dimensional topological insulators (TIs), the nontrivial topology of their electronic bands causes a gapless state on the solid surfaces ${ }^{1-12}$, which is associated with forbidden backscattering, spin helicity, and its possible application in lowdissipation electronics. Dissipationless TI edge devices based on the quantum anomalous Hall ${ }^{13-16}(\mathrm{QAH})$ effect and the quantum Hall $^{17-20}(\mathrm{QH})$ effect have been reported with clear quantization plateaus spaced apart by $e^{2} / h$, where $e$ is the electron charge and $h$ is the Planck constant. $\mathrm{QH}$ devices have been demonstrated to operate at more tolerable temperatures of almost $50 \mathrm{~K}$, in which the surface electronics operate in a dimension among the $(-3 / 2$, $-3 / 2) \sim(3 / 2,5 / 2)$ spaces. Protected by time-reversal symmetry, practical TI nanodevices have a pair of parallel-transport carrier states on their two surfaces. However, due to the no-go theorem $^{21,22}$, the two topological surface states (TSSs) always appear as a pair and are expected to quantize synchronously.

Theoretically, breaking the time-reversal symmetry of a single Dirac fermion gives rise to a mass. The massive Dirac fermion has been predicted to exhibit a parity anomaly $2,23,24$ in high energy physics, although this phenomenon has yet to be observed experimentally despite being studied at some length in theoretical terms. The term "parity anomaly" implies that an infinitesimal mass will inevitably break the parity symmetry, resulting in a half-QH effect, which in turn means a half- $\mathrm{QH}$ conductance $(1 / 2$ $\left.e^{2} / h\right)$. The TSS whose low-energy effective spectrum mimics the Dirac fermion provides a perfect platform for achieving the parity anomaly. However, asynchrony quantization has still not been achieved to date, and the quantization trajectory of a single surface has not yet been studied.

Here we describe the deposition of Co clusters on bulkinsulating $\mathrm{BiSbTeSe}_{2}$ devices, which delays the Hall quantization of the top surface. An interesting quasi-half-integer plateau (-3/2 $\left.e^{2} / h\right)$ appears at -7.2 Tesla $(\mathrm{T})$. Renormalization group flow diagrams ${ }^{14,25}$ (RGFDs) allow extraction of two sets of converging points (CVPs) in the $\left(\sigma_{\mathrm{xy}}, \sigma_{\mathrm{xx}}\right)$ space, where the top surface exhibits an anomalous quantization trajectory, while the bottom surface retains the $1 / 2$ quantization. This effect can be interpreted using a delayed Landau level (LL) hybridization (DLLH) model, in which the Co clusters induce a sizeable Zeeman gap ( $>$ $4.8 \mathrm{meV}$ ) through antiferromagnetic exchange coupling on the top surface.

\section{Results}

The QH effect of the surface-dominated TI. We fabricated fieldeffect-transistor BSTS ${ }^{10}$ devices using a standard lift-off procedure (see "Methods" section). The Co clusters were deposited on the bare devices using a cluster beam source as described elsewhere ${ }^{26}$. A schematic of our devices is shown in the inset of Fig. 1a. Elemental energy-dispersive X-ray spectroscopy was used to confirm the successful deposition of the clusters. We measured the sample both before and after Co cluster deposition; we refer to the sample after Co deposition as sample A, and the sample before Co deposition as sample $\mathrm{A}^{\prime}$. Figure 1a also indicates the typical temperature (T)-dependent resistance of sample A, showing insulating behavior at high temperatures (above $100 \mathrm{~K}$ ), which is attributed to the transport of the bulk electronic state, followed by a metallic increase at low temperatures. This is similar to the case for pristine BSTS as reported by other groups ${ }^{11,17}$. The pristine bulk maximum resistivity reached $>10$ $\Omega \mathrm{cm}$. An atomic force microscopic image (Fig. 1b) shows small islands with diameters of a few tens of nanometers and heights of

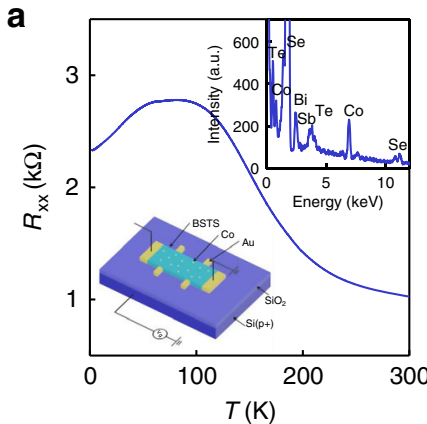

b

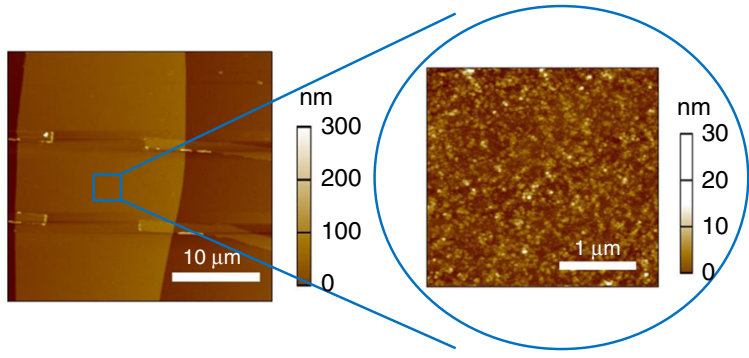

C

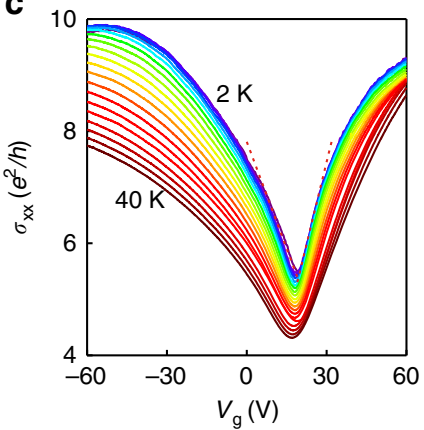

d

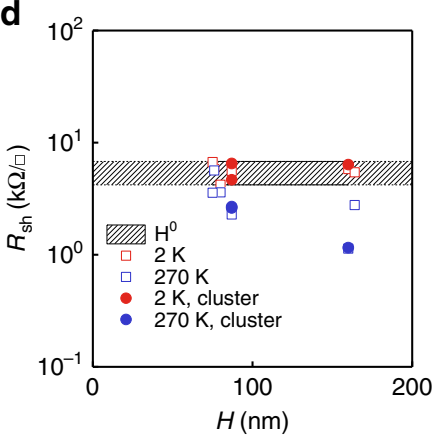

e

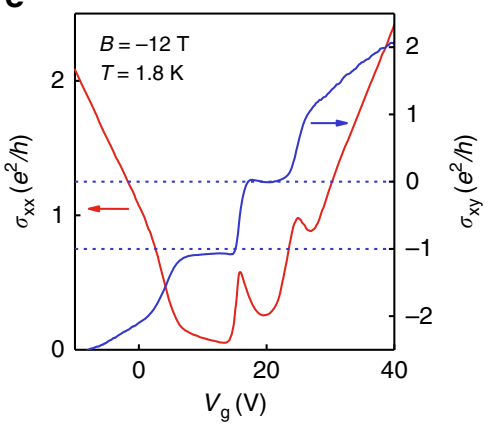

Fig. 1 Surface dominant transport and quantum Hall effect in the BSTS device. a A typical curve of temperature-dependent resistance. The inset shows the backgate measurement configuration and the elemental composition of the sample. $\mathbf{b}$ Atomic force microscopic image of the device. The zoom-in of (b) shows the granular morphology of the sample surface. The sizes of the clusters are in the tens of nanometers and their heights are about $5 \mathrm{~nm}$. The scale bar on the left is $10 \mu \mathrm{m}(1 \mu \mathrm{m}$ in the zoom-in). c Gate-dependent conductivity from 2 to $40 \mathrm{~K}$ with a step of $2 \mathrm{~K}$, exhibiting the bipolar characteristic. The minimum conductivity appears at a gate voltage of around $16.6 \mathrm{~V}$. $\mathbf{d}$ The sheet resistance of several samples (some of them measured before the Co cluster deposition). Red dots are the data measured at $2 \mathrm{~K}$, all of which fall within a small range, with or without Co clusters. This indicates the dominance of the surface transport. The blue dots, measured at $270 \mathrm{~K}$, fall outside the shadowed regime, however. $H$ is the height of the samples, and $H^{0}$ denotes the zeroth power of $H$. e Quantum Hall effect observed at a temperature of $1.8 \mathrm{~K}$ and a field of $-12 \mathrm{~T}$ 
a
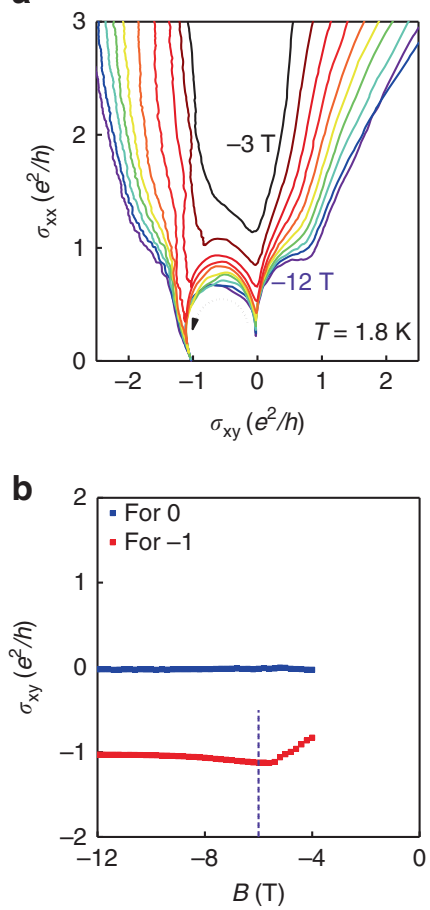

c
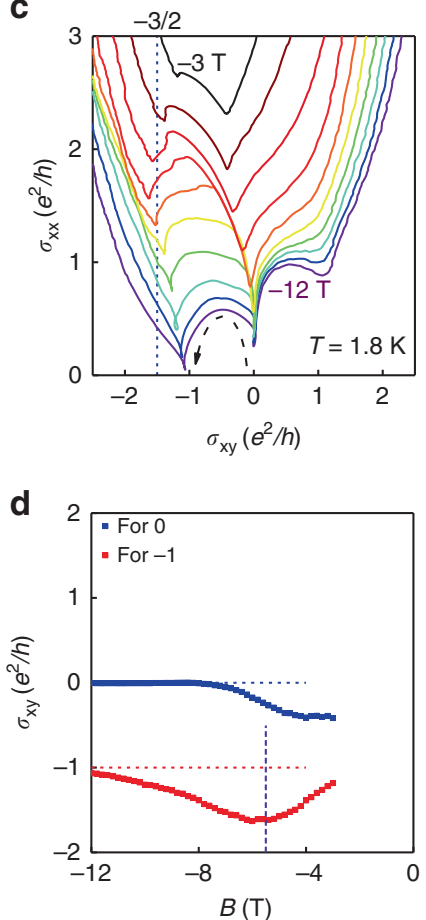

e
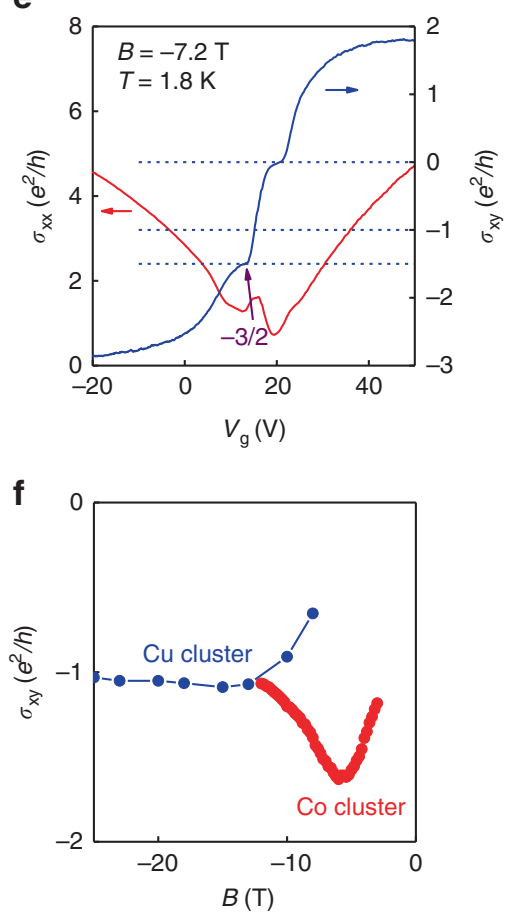

Fig. 2 Renormalization group flow diagram analysis with the result of quantization trajectory of single Dirac channel. a Renormalization group flow diagram (RGFD) analysis in $\left(\sigma_{\mathrm{xy}}, \sigma_{\mathrm{xx}}\right)$ space based on the data measured between -3 and $-12 \mathrm{~T}$ with the step being $-1 \mathrm{~T}$ for the sample before the Co deposition (sample $\mathrm{A}^{\prime}$ ) at $1.8 \mathrm{~K}$. The converging points (CVPs), the local minimum with a vanishing $\sigma_{\mathrm{xx}}$ and plateau $\sigma_{\mathrm{xy}}$ indicate complete quantum Hall (QH) filling. $\mathbf{b}$ We note two sets of CVPs, pointing to $\sigma_{\mathrm{xy}}$ of 0 and $-e^{2} / h$. The dashed line marks the $\sigma_{\mathrm{xy}}$ minimum. $\mathbf{c}$ RGFD analysis in ( $\sigma_{\mathrm{xy}}, \sigma_{\mathrm{xx}}$ ) space based on the data measured between -3 and $-12 \mathrm{~T}$ with a step of $-1 \mathrm{~T}$ for the Co-decorated sample (sample A). Note the anomalous RGFD trajectory is characteristic in this system. d The CVP trajectories for sample A. The converging trajectories are clearly different. The dashed line marks the $\sigma_{\mathrm{xy}}$ minimum. e The $3 / 2$ QH plateau observed at $-7 \mathrm{~T}$ and $1.8 \mathrm{~K}$, while scanning the gate voltage. $\mathbf{f}$ The CVPs of the -1 plateau for the Cu clusters decorated BSTS (sample D) and the Co clusters decorated BSTS (sample A). Sample D does not show the anomalous trajectory of sample A

around $5 \mathrm{~nm}$. We measured several samples, and we list all the transport parameters of the devices in Supplementary Table 1.

In Fig. 1c, we show the backgate voltage $\left(V_{\mathrm{g}}\right)$-dependent longitudinal conductivity $\left(\sigma_{\mathrm{xx}}\right)$ of sample A measured at a series of temperatures from 2 to $40 \mathrm{~K}$ at intervals of $2 \mathrm{~K}$. All the transfer curves show bipolar transport behaviors, where the minimum conductivity corresponds to the Dirac point (DP) of the bottom surface. At $T=2 \mathrm{~K}$, the $\mathrm{DP}$ is around $V_{\mathrm{g}}=16.6 \mathrm{~V}$ and the minimum conductivity is about $5 e^{2} / h$. Furthermore, $\sigma_{\mathrm{xx}}$ exhibits a linear dependence on gate voltage ${ }^{18,27}$. We measured several samples, including some prior to cluster deposition. We show the sheet resistance $\left(R_{\mathrm{sh}}\right)$ of all of these at $2 \mathrm{~K}$ (red dots) and $270 \mathrm{~K}$ (blue dots) in Fig. 1d, where we find that all the values of $R_{\mathrm{sh}}$ at $2 \mathrm{~K}$ fall into a small range independent of their thickness. Here, $H$ is the height of the samples. This implies surface dominant transport in our devices, allowing successful observation of the $\mathrm{QH}$ effect.

High magnetic field drives BSTS to the QH state. In Fig. 1e, at a magnetic field $(B)$ of $12 \mathrm{~T}$, the Hall conductivity $\left(\sigma_{\mathrm{xy}}\right)$ of sample A steadily reaches zero and $-e^{2} / h$ while scanning the backgate voltage from 0 to $40 \mathrm{~V}$. In the meantime, the longitudinal conductivity $\left(\sigma_{\mathrm{xx}}\right)$ fluctuates and shows local minimums at both Hall plateaus. Similar QH effects ${ }^{17-20}$ have been interpreted based on the fact that the two TSSs (top and bottom) each contribute a half-integer plateau. The DP for the bottom surface is $V_{\mathrm{g}}=16.6 \mathrm{~V}$ (Supplementary Table 1). Hence, we assign a half-integer index $\nu$ for each surface for both plateaus ${ }^{17,18,20} \cdot \nu_{\mathrm{b}}$ (bottom surface) is $1 / 2$ and $-1 / 2$, respectively, and $\nu_{\mathrm{t}}$ (top surface) is maintained at $-1 / 2$. The top surface is p-type transport.
The anomalous quantization trajectory extracted by RGFD. For graphene, integer $\mathrm{QH}$ plateaus of series $(4 n+2) e^{2} / h$ have been observed 28,29 , due to the spin and sublattice degeneracy of the material ${ }^{30-32}$. $\mathrm{QH}$ experiments on TI devices suffer from parallel transport of the two surface states and result in $\mathrm{QH}$ plateaus of $\left(n_{\mathrm{T}}+n_{\mathrm{B}}+1\right) e^{2} / h$. However, in our cluster-decorated device it is interesting to note that the quasi-half-integer $-3 / 2$ Hall plateau appears when measuring at a medium field of $-7 \mathrm{~T}$, as marked by the purple arrow in Fig. $2 \mathrm{e}$, in which a zero plateau is also seen. Both plateaus are supported by fixed $\sigma_{\mathrm{xy}}$ values and local minimums of $\sigma_{\mathrm{xx}}$. We prepared three different samples. Measurements made on two other devices also show $\mathrm{QH}$ plateaus near $-1 / 2$ and $-3 / 2 e^{2} / h$ (Supplementary Fig. 3 and Supplementary Note 2). The reproducibility for different samples indicates that the quasi-half-integer plateau should be independent of both the parameter windows and the details of the samples. These observations lead to the question: Is this quasi-half-QH plateau an indication of the Landau quantization of a separated Dirac channel $^{2}$ ? In the following, we devote our efforts to serious analysis of the nature of the half-integer plateau by extracting the RGFD of the corresponding state.

We note in Fig. 1e that when the magnetic field is increased to $-12 \mathrm{~T}$, the plateau of the sample becomes $n=-1$, corresponding to the quantization of both surfaces, $\nu_{\mathrm{b}}=-1 / 2$ and $\nu_{\mathrm{t}}=-1 / 2$. For the $n=0$ state, $\nu_{\mathrm{b}}=1 / 2$ and $\nu_{\mathrm{t}}=-1 / 2$ is well maintained ${ }^{17,20}$. The context is better explained by sweeping the backgate voltage at a fixed magnetic field. RGFD analysis was carried out in the $\left(\sigma_{\mathrm{xx}}\right.$, $\sigma_{\mathrm{xy}}$ ) space before (Fig. 2a, b) and after cluster deposition (Fig. 2c, d). The CVPs of the RGFD indicate complete Landau 
a

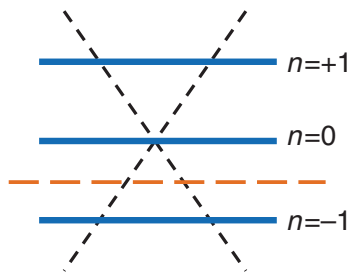

C

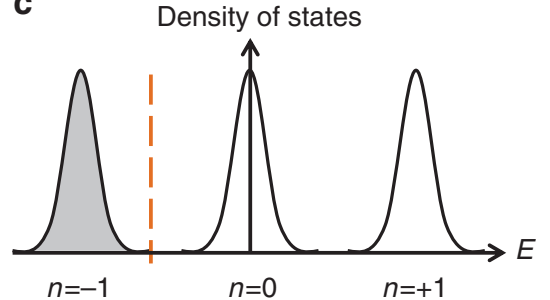

e

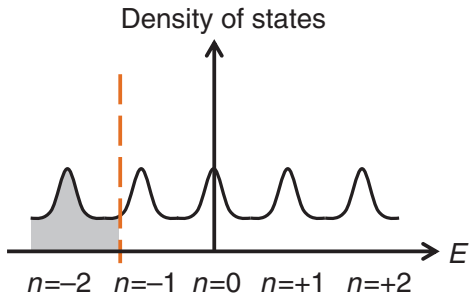

b
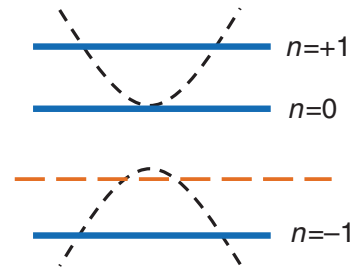

d Density of states

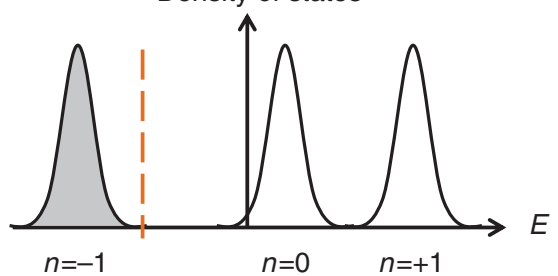

$\mathbf{f}$

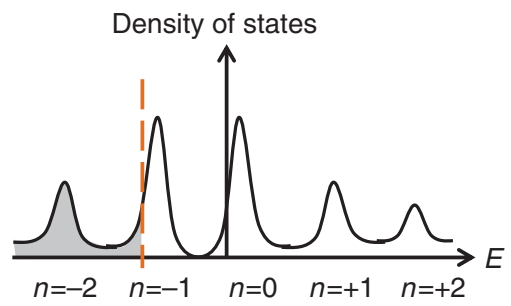

Fig. 3 The delayed Landau level hybridization model. a, c, e and $\mathbf{b}, \mathbf{d}, \mathbf{f}$ schematically show the Landau Level (LL) diagram before/after Co cluster deposition, respectively, during Hall quantization. The orange dashed lines indicate the Fermi energy. Under a magnetic field, the deposited Co clusters induce a Zeeman-like gap in the Dirac cone of the top surface. The zeroth LL is shifted to the top of the Zeeman gap (b), while the bottom surface is unchanged, similar to the case for clean devices (a). This makes no significant difference at a sufficiently high field (c, d). At a medium field, it leads to the observed anomalous quantization trajectory (e, f). The anomalous RGFD trajectory (Fig. 2c) is the result of the delayed LL hybridization (DLLH) of the top surface (f)

quantization of the bottom surface. Two sets of CVPs can be seen with $\sigma_{\mathrm{xy}}$ pointing to 0 and $-e^{2} / h$, respectively. Clearly, both sets of CVPs show continuous evolution and finally reach an integer with increasing field. Note that in all states of the CVPs in RGFD, the bottom surfaces have been quantized. In the whole parameter region, it is the deposited top surface that demonstrates a continuous evolution of Hall conductivity. Therefore, the $-3 / 2$ $\mathrm{QH}$ plateau should be the result of the sum of two contributions, with one being the $-1 / 2$ quantized bottom surface and the other being the $-e^{2} / h$ Hall conductivity from the top surface.

In Fig. 2d, we plot the two sets of CVPs as curves, which are essentially the quantization trajectories of the top surfaces after subtracting a $-1 / 2$ (or 1/2) integer from the $\mathrm{QH}$ index of the bottom surfaces ${ }^{17}$. This way, we can extract the quantization trajectory of a separate Dirac channel. As shown in Fig. 2d, the quantization of the -1 plateau travels along an anomalous trajectory over a large range of nearly one $e^{2} / h$, which is not seen in the undecorated BSTS (Fig. 2b). We repeated these measurements in two other samples, where the quantization trajectories were anomalous (Supplementary Fig. 3 and Supplementary Note 2) and reproduced in both cases. Being sample-independent, we expect such an anomalous quantization trajectory to be a characteristic of our Co-cluster-decorated BSTS samples.

\section{Discussion}

We propose a DLLH model to interpret the anomalous LL quantization trajectory in Co-cluster-decorated BSTS, in which two effects are taken into account. First, the Co clusters form some magnetic moments with finite magnetization strengths $M_{\mathrm{z}}(B)$ under a field. The magnetic moments of the Co clusters further polarize the Dirac fermions on the top surface through antiferromagnetic coupling, which results in a sizeable Zeeman gap $^{19,33} m=-J M_{\mathrm{Z}} / 2 \mu_{\mathrm{B}}$, where $J$ is the exchange interaction constant and $\mu_{\mathrm{B}}$ is the Bohr magneton. Second, a medium field enlarges the Zeeman gap and forms the LL quantization. Solving the Dirac equation with a gauge field (Supplementary Note 3 and $4)$, we obtain an unexpected zeroth LL with energy $E_{0}=-\operatorname{sgn}(B)$ $M_{\mathrm{Z}} / 2 \mu_{\mathrm{B}}$, which is positioned precisely at the top of the Zeeman gap. Such a magnetization condition is reasonable in our sample. Here the magnetization of the top surface increases with the field, therefore it is hard to detect directly and hard to see any loops. However, we may check its effect by fitting a weak antilocalization using the Hikami-Larkin-Nagaoka formula. We find that the channel parameter decreases when we increase the fitting range of the magnetic field from $(0-0.2 \mathrm{~T})$ to $(0-1 \mathrm{~T})$. Such a decrease can be related to the contribution of weak localization from a magnetization gap of several meV.

We begin the interpretation by considering an undecorated sample in a sufficiently high field, where conventional Landau quantization is achieved in Dirac fermions (Fig. 3a, c). When the decreasing field reaches a threshold value, the LL spacings $d_{\mathrm{L}}$ gradually hybridize with each other (Fig. 3e), resulting in a smooth evolution of $\sigma_{\mathrm{xy}}$ from $-e^{2} / h$ towards zero, as shown by the red curve in Fig. 2b. The threshold field can be interpreted from the position of the dashed line in Fig. $2 \mathrm{~d}$, with $B_{1}=-6 \mathrm{~T}$. The two effects discussed above take place after Co cluster deposition. The resulting shifted zeroth LL leads to an increase in the distance between -1 and 0 LL by $E_{0}$. At a sufficiently high field, we can expect no significant difference between the pristine (Fig. $3 \mathrm{c}$ ) and the deposited sample (Fig. $3 \mathrm{~d}$ ) because the LL spacing $d_{\mathrm{L}}$ is much larger than the Zeeman gap (Fig. 3b). This accounts for the perfect quantization at high field for both samples. However, at a 
lower field where the LL hybridization begins in the pristine case, the enlarged LL spacing $E_{0}$ would be comparable to that in the LL spacing in the pristine sample. This results in the DLLH and leads to the following key difference between the two cases. In the pristine sample, Fig. $2 \mathrm{~b}$ indicates that the -1 LL hybridizes before it crosses the Fermi energy. In the process of lowering the field, the electrons can still fill up all the extension states in the $-1 \mathrm{LL}$, therefore no significant decrease in Hall conductivity is observed. However, in the decorated sample, hole states emerge in the -1 LL levels before hybridization, giving rise to the decrease of $\sigma_{\mathrm{xy}}$ before it evolves toward zero.

This is the experimental case, illustrating the physics of the anomalous quantization trajectory in RGFD (Fig. 2d). Around $B$ $=-7 \mathrm{~T}$, the Hall conductivity from the bottom surface is still quantized at $-1 / 2 e^{2} / h$ (Fig. $2 b$ ), while the top surface is nonquantized and exhibits Hall conductivity traveling along the anomalous trajectory due to the DLLH effect. Together with other effects such as the lateral cross section, the anomalous quantization trajectory leads to the intangible Hall plateaus observed in Fig. 2c, d. Finally, we note the results of some scanning tunneling microscopy experiments on undecorated $\mathrm{TIs}^{34}$, which show clear Zeeman shifts of the zeroth LL amounting to a few meV. However, this effect depends on the material. We find it appropriate to neglect the Zeeman effect for the undecorated case here (Supplementary Note 5).

On the basis of the above model, we estimate the width of the LLs as well as the Zeeman gap due to magnetic doping. It is known that the LLs begin to hybridize when their spacing becomes comparable to the width. Using this condition, we can estimate the LL width for the undecorated sample as $w=$ $25.8 \mathrm{meV}$, by the critical field $(-5.6 \mathrm{~T})$ in the quantization trajectory (dashed line marked on the red curve in Fig. 2b). This broadening of the LL is related to the electronic inhomogeneity, which is most likely to originate from the random configuration of atoms in this material ${ }^{35-37}$. Figure $2 \mathrm{~d}$ shows that the Hall conductivity begins to decrease from -1 when the field is reduced from $12 \mathrm{~T}$, indicating that the electron gradually fills into the extension region in the $n=-1 \mathrm{LL}$ in this process. From $B=-12$ to $-6 \mathrm{~T}$, the Hall conductivity drops from -1 to $-1.7 e^{2} / h$, until which point the LL hybridization leads to an upturn in the Hall conductivity. We can use this observation to estimate the width of the extension region to be $d \approx 15.7 \mathrm{meV}$. Again using the condition for hybridization, we obtain $w^{\prime}=m+26.6 \mathrm{meV}$, where $w^{\prime}$ is the LL width after deposition and $m=J \operatorname{sgn}(B) M_{\mathrm{Z}} / 2 \mu_{\mathrm{B}}$ is the Zeeman gap. Here the critical field moves to around $-6 \mathrm{~T}$ (dashed line marked on the red curve in Fig. 2d) due to the delay effect and the broadened LLs. Usually, the extension region is much smaller than the width of the LL. As an estimate, we can safely assume that $d<0.5 w^{\prime}$, and therefore $w^{\prime}>31.4 \mathrm{meV}$, such that $m$ $>4.8 \mathrm{meV}$. This gives the lower bound for the estimated Zeeman gap.

To lend further weight to these results, we measured the sample with $\mathrm{Cu}$ cluster deposition. Different from the Co clusters studied here, the sample with a non-magnetic $\mathrm{Cu}$ cluster (sample D) does not exhibit an anomalous quantization trajectory (Fig. 2f); instead it shows a normal trajectory similar to the undecorated sample (Fig. 2a, b). For comparison, the CVPs for the Co-cluster-decorated sample (sample A) are plotted in Fig. 2f. The difference between $\mathrm{Cu}$ and Co cluster decoration can clearly be seen. We note that the non-magnetic clusters do not bring the effect to the QH state of the TI as the magnetic clusters do. The CVP trajectories of all the samples we measured are shown in Supplementary Table 1 . The RGFD of sample D is shown in Supplementary Fig. 1 (Supplementary Note 1). The separate quantization of the top/bottom surfaces is thus further corroborated by the distinct LL widths. Nevertheless, the greatly disordered top channels still accommodate the QH transport, reflecting the priority of the spin-helical Dirac electrons.

\section{Methods}

Crystal growth and device fabrication. Well-refined BSTS crystals were grown by melting high-purity elements of $\mathrm{Bi}, \mathrm{Sb}$, Te, Se with a molar ratio of $1: 1: 1: 2$ at $850^{\circ} \mathrm{C}$ for $24 \mathrm{~h}$ in evacuated quartz tubes, followed by cooling to room temperature over 1 week. The crystal has a low bulk carrier density, and can be cleaved easily. Following the work process of graphene, the BSTS microflakes were exfoliated and transferred on doped $\mathrm{Si}$ substrates coated with $300 \mathrm{~nm} \mathrm{SiO}$. Au electrodes were applied by standard electron-beam lithography and electron beam evaporation. The Co clusters were deposited by a cluster beam system as described elsewhere ${ }^{26}$. The thickness of the samples and the clusters were measured using an atomic force microscope.

Transport measurement and data analysis. Transport measurements were performed at low temperatures down to $1.8 \mathrm{~K}$ with a magnetic field up to $12 \mathrm{~T}$ Standard lock-in amplifiers (Stanford Research System SR830) with a lowfrequency $(<20 \mathrm{~Hz})$ excitation current of $200 \mathrm{nA}$ (Keithley 6221) were used. The high magnetic field experiments were performed at the High Magnetic Field Laboratory, Chinese Academy of Sciences. The sheet resistance was $R_{\mathrm{sh}}=R_{\mathrm{xx}}(W / L)$ $=\rho_{\mathrm{xx}}$, where $W$ and $L$ are respectively the channel width and length between the longitudinal magnetoresistance $R_{\mathrm{xx}}$ voltage probes. For the Hall effect, $R_{\mathrm{xy}}=\rho_{\mathrm{xy}}$. The conductivities are $\sigma_{\mathrm{xx}}=\rho_{\mathrm{xx}} /\left(\rho_{\mathrm{xx}}{ }^{2}+\rho_{\mathrm{xy}}{ }^{2}\right)$ and $\sigma_{\mathrm{xy}}=\rho_{\mathrm{xy}} /\left(\rho_{\mathrm{xx}}^{2}+\rho_{\mathrm{xy}}^{2}\right) . \sigma_{\mathrm{xy}}$ and $\sigma_{\mathrm{xx}}$ are dependent on the parameters, such as temperature, magnetic field and gate voltage. The RGFDs are plotted with $\sigma_{\mathrm{xy}}$ on the abscissa and $\sigma_{\mathrm{xx}}$ on the ordinate in the $\left(\sigma_{\mathrm{xy}}, \sigma_{\mathrm{xx}}\right)$ space. Here the temperature is unchanged, and the RGFDs are plotted with magnetic field for backgate voltage. Each curve in the RGFDs is in a fixed magnetic field, such as $-12 \mathrm{~T}$, so every point in the $\left(\sigma_{\mathrm{xy}}, \sigma_{\mathrm{xx}}\right)$ space corresponds to a backgate voltage, which is actually the Fermi level.

Data availability. The data in this work are available from the corresponding author on reasonable request.

Received: 2 February 2017 Accepted: 15 August 2017

Published online: 17 October 2017

\section{References}

1. Hasan, M. Z. \& Kane, C. L. Colloquium: Topological insulators. Rev. Mod. Phys 82, 3045-3067 (2010).

2. Qi, X.-L. \& Zhang, S.-C. Topological insulators and superconductors. Rev. Mod. Phys. 83, 1057-1110 (2011)

3. Kong, D. et al. Ambipolar field effect in the ternary topological insulator $\left(\mathrm{Bi}_{\mathrm{x}} \mathrm{Sb}_{1-\mathrm{x}}\right)_{2} \mathrm{Te}_{3}$ by composition tuning. Nat. Nanotech. 6, 705-709 (2011).

4. Fu, L., Kane, C. L. \& Mele, E. J. Topological insulators in three dimensions. Phys. Rev. Lett. 98, 106803 (2007).

5. Young, A. F. et al. Tunable symmetry breaking and helical edge transport in a graphene quantum spin Hall state. Nature 505, 528-532 (2014).

6. Bardarson, J. H. \& Moore, J. E. Quantum interference and Aharonov-Bohm oscillations in topological insulators. Rep. Prog. Phys. 76, 056501 (2013).

7. Chen, Y. L. et al. Experimental realization of a three-dimensional topological insulator, $\mathrm{Bi}_{2} \mathrm{Te}_{3}$. Science 325, 178-181 (2009).

8. Hsieh, D. et al. A tunable topological insulator in the spin helical Dirac transport regime. Nature 460, 1101-1105 (2009).

9. Qi, X.-L., Hughes, T. L. \& Zhang, S.-C. Topological field theory of time-reversal invariant insulators. Phys. Rev. B 78, 195424 (2008).

10. Arakane, T. et al. Tunable Dirac cone in the topological insulator $\mathrm{Bi}_{2-\mathrm{x}} \mathrm{Sb}_{\mathrm{x}} \mathrm{Te}_{3}$ ${ }_{\mathrm{y}} \mathrm{Se}_{\mathrm{y}}$. Nat. Commun. 3, 636 (2012).

11. Brahlek, M., Koirala, N., Salehi, M., Bansal, N. \& Oh, S. Emergence of decoupled surface transport channels in bulk insulating $\mathrm{Bi}_{2} \mathrm{Se}_{3}$ thin films. Phys. Rev. Lett. 113, 026801 (2014).

12. Chen, T. et al. Topological transport and atomic tunnelling-clustering dynamics for aged $\mathrm{Cu}$-doped $\mathrm{Bi}_{2} \mathrm{Te}_{3}$ crystals. Nat. Commun. 5, 5022 (2014).

13. Chang, C.-Z. et al. Experimental observation of the quantum anomalous hall effect in a magnetic topological insulator. Science 340, 167-170 (2013).

14. Checkelsky, J. G. et al. Trajectory of the anomalous Hall effect towards the quantized state in a ferromagnetic topological insulator. Nat. Phys. 10, 731 (2014).

15. Kou, X. et al. Scale-invariant quantum anomalous Hall effect in magnetic topological insulators beyond the two-dimensional limit. Phys. Rev. Lett. 113 137201 (2014).

16. Chang, C. Z. et al. High-precision realization of robust quantum anomalous Hall state in a hard ferromagnetic topological insulator. Nat. Mater. 14, 473-477 (2015). 
17. Xu, Y. et al. Observation of topological surface state quantum Hall effect in an intrinsic three-dimensional topological insulator. Nat. Phys. 10, 956-963 (2014).

18. Yoshimi, R. et al. Quantum Hall effect on top and bottom surface states of topological insulator $\left(\mathrm{Bi}_{1-\mathrm{x}} \mathrm{Sb}_{\mathrm{x}}\right)_{2} \mathrm{Te}_{3}$ films. Nat. Commun. 6, 6627 (2015).

19. Yoshimi, R. et al. Quantum Hall states stabilized in semi-magnetic bilayers of topological insulators. Nat. Commun. 6, 8530 (2015).

20. Xu, Y., Miotkowski, I. \& Chen, Y. P. Quantum transport of two-species Dirac fermions in dual-gated three-dimensional topological insulators. Nat. Commun. 7,11434 (2016).

21. Nielsen, H. B. \& Ninomiya, M. A no-go theorem for regularizing chiral fermions. Phys. Lett. B. 105, 219-223 (1981).

22. Morimoto, T., Furusaki, A. \& Nagaosa, N. Charge and spin transport in edge channels of a $\nu=0$ quantum Hall system on the surface of topological insulators. Phys. Rev. Lett. 114, 146803 (2015).

23. Semenoff, G. W. Condensed-matter simulation of a three-dimensional anomaly. Phys. Rev. Lett. 53, 2449-2452 (1984).

24. Redlich, A. N. Gauge noninvariance and parity nonconservation of threedimensional fermions. Phys. Rev. Lett. 52, 18-21 (1984).

25. Khmelnitskii, D. E. Quantization of Hall conductivity. Jetp Lett. 38, 552-556 (1983).

26. Song, F. et al. Visualizing plasmon coupling in closely spaced chains of Ag nanoparticles by electron energy-loss spectroscopy. Small. 6, 446-451 (2010).

27. Dean, C. R. et al. Boron nitride substrates for high-quality graphene electronics. Nat. Nanotechol. 5, 722-726 (2010).

28. Novoselov, K. S. et al. Two-dimensional gas of massless Dirac fermions in graphene. Nature 438, 197-200 (2005).

29. Zhang, Y., Tan, Y. W., Stormer, H. L. \& Kim, P. Experimental observation of the quantum Hall effect and Berry's phase in graphene. Nature 438, 201-204 (2005).

30. Young, A. F. et al. Spin and valley quantum Hall ferromagnetism in graphene. Nat. Phys. 8, 550-556 (2012).

31. Zhang, Y. et al. Landau-level splitting in graphene in high magnetic fields. Phys. Rev. Lett. 96, 136806 (2006).

32. Bao, W. et al. Stacking-dependent band gap and quantum transport in trilayer graphene. Nat. Phys. 7, 948-952 (2011).

33. Jiang, Y. et al. Mass acquisition of Dirac fermions in magnetically doped topological insulator $\mathrm{Sb}_{2} \mathrm{Te}_{3}$ films. Phys. Rev. B 92, 195418 (2015).

34. Fu, Y. S. et al. Observation of Zeeman effect in topological surface state with distinct material dependence. Nat. Commun. 7, 10829 (2016).

35. Ren, Z., Taskin, A. A., Sasaki, S., Segawa, K. \& Ando, Y. Optimizing $\mathrm{Bi}_{2-\mathrm{x}} \mathrm{Sb}_{\mathrm{x}} \mathrm{Te}_{3}$ ${ }_{-y} \mathrm{Se}_{\mathrm{y}}$ solid solutions to approach the intrinsic topological insulator regime. Phys. Rev. B 84, 165311 (2011).

36. Kim, S. et al. Robust protection from backscattering in the topological insulator $\mathrm{Bi}_{1.5} \mathrm{Sb}_{0.5} \mathrm{Te}_{1.7} \mathrm{Se}_{1.3}$. Phys. Rev. Lett. 112, 136802 (2014).

37. Beidenkopf, $H$. et al. Spatial fluctuations of helical Dirac fermions on the surface of topological insulators. Nat. Phys. 7, 939-943 (2011).

\section{Acknowledgements}

We acknowledge the discussions with Professor Yong P. Chen at Purdue University and Professor Qianghua Wang, Xiangang Wan at Nanjing University. We also thank the National Key P\&D Program of China (Grants No. 2017YFA0303203), the National Key Projects for Basic Research in China (Grant No: 2013CB922103), the National Natural Science Foundation of China (Grant Nos: U1732273, 91421109, 91622115, 11522432, 11574217, 61176088 and 11274003), the NSF of Jiangsu Province (Grant BK20160659 and BK20130054), the Fundamental Research Funds for the Central Universities (020414380056 and 020414380057) and the Opening Project of Wuhan National High Magnetic Field Center. We would also like to acknowledge the assistance of the Nanofabrication and Characterization Center at the Physics College of Nanjing University.

\section{Author contributions}

F.S.: Conceived the work. X.-C.P.: Prepared the bulk crystal. S.Z. and Z.B.: Fabricated the devices. Z.W. and M.W.: Undertook the cluster deposition. F.F.: Made the energydispersive spectroscopy. J.Z., C.X. and L.P.: Provided the conditions of high magnetic field experiment. S.Z.: Performed the transport measurement. R.W. and B.W.: Proposed the theoretical model. S.Z., R.W. and F.S.: Co-wrote the paper. G.Y., J.L., Y.L., X.W., Y.Z., B.W., D.X. and G.W.: Participated in the discussion. All authors commented on the manuscript.

\section{Additional information}

Supplementary Information accompanies this paper at doi:10.1038/s41467-017-01065-7.

Competing interests: The authors declare no competing financial interests.

Reprints and permission information is available online at http://npg.nature.com/ reprintsandpermissions/

Publisher's note: Springer Nature remains neutral with regard to jurisdictional claims in published maps and institutional affiliations.

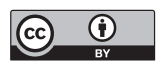

Open Access This article is licensed under a Creative Commons Attribution 4.0 International License, which permits use, sharing, adaptation, distribution and reproduction in any medium or format, as long as you give appropriate credit to the original author(s) and the source, provide a link to the Creative Commons license, and indicate if changes were made. The images or other third party material in this article are included in the article's Creative Commons license, unless indicated otherwise in a credit line to the material. If material is not included in the article's Creative Commons license and your intended use is not permitted by statutory regulation or exceeds the permitted use, you will need to obtain permission directly from the copyright holder. To view a copy of this license, visit http://creativecommons.org/ licenses/by/4.0/

(C) The Author(s) 2017 